\title{
Lumen
}

Selected Proceedings from the Canadian Society for Eighteenth-Century Studies

\section{Informer et diffuser la pensée dans la France du dernier siècle de l'Ancien Régime}

\section{François Moureau}

Volume 28, 2009

URI : https://id.erudit.org/iderudit/1012036ar

DOI : https://doi.org/10.7202/1012036ar

Aller au sommaire du numéro

Éditeur(s)

Canadian Society for Eighteenth-Century Studies / Société canadienne d'étude du dix-huitième siècle

ISSN

1209-3696 (imprimé)

1927-8284 (numérique)

Découvrir la revue

Citer cet article

Moureau, F. (2009). Informer et diffuser la pensée dans la France du dernier siècle de l'Ancien Régime. Lumen, 28, 29-50. https://doi.org/10.7202/1012036ar

Copyright (c) Canadian Society for Eighteenth-Century Studies / Sociéte canadienne d'étude du dix-huitième siècle, 2009
Ce document est protégé par la loi sur le droit d'auteur. L'utilisation des services d'Érudit (y compris la reproduction) est assujettie à sa politique d'utilisation que vous pouvez consulter en ligne.

https://apropos.erudit.org/fr/usagers/politique-dutilisation/ 


\section{$2:$ Informer et diffuser la pensée dans la France du dernier siècle de l'Ancien Régime}

Dans notre siècle, la communication est quasi instantanée et planétaire : c'est une expérience nouvelle qui ne doit pas faire oublier que le monde a vécu pendant des millénaires sans le moindre medium de masse. Les « annales » historiques brochées sur des récits « mythiques » racontaient les grandes époques des peuples : épopée de Gilgamesh, Pentateuque, matière troyenne $\mathrm{d}^{\prime}$ Homère à Virgile ${ }^{1}$. Puis vinrent les historiens, d'abord au service des princes et de leur gloire, puis des nations impériales. Fussent-elles réalisées au plus près des événements, ces fresques étaient là pour durer. La presse est fondée sur le transitoire, le périssable ; elle ne dit pas l'histoire, elle porte un regard de myope sur le monde. C'est un produit jetable de la modernité, de l'alphabétisation des élites, voire des masses, et le fruit singulier de la technologie du reproductible.

Apparu au milieu du $X V^{e}$ siècle en Occident $l^{\prime}$ «ars artificialiter scribendi », appelé vulgairement imprimerie, devança de plus d'un siècle et demi le premier « journal » d'information, certes précédé par une floraison de brochures dites « canards » ou « occasionnels $»^{2}$. Le monde

1 Voir dans les Questions sur l'Encyclopédie de Voltaire, l'article «ANNALES » qui en fait l'analyse critique. Nous l'avons édité dans les CEuvres complètes, t. 38 : Questions sur l'Encyclopédie, par des amateurs. II : A-Aristée, Oxford, Voltaire Foundation, 2007, p. 385-389.

2 Jean-Pierre Seguin, L'Information en France avant le périodique. 517 canards imprimés entre 1529 et 1631, Paris, G.-P. Maisonneuve et Larose, 1964 ; Jean-Pierre Vittu, art. "CANARD ", dans Pascal Fouché, Daniel Péchoin et Philippe Schuwer (sous la direction de), Dictionnaire encyclopédique du livre, Paris, Éditions du Cercle de la Librairie , 2002, t. I, p. 435. 
de la communication imprimée fut dès l'origine l'objet de la suspicion des pouvoirs politiques et religieux: en France, dépôt légal dès Fran-

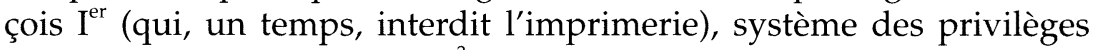
$\mathrm{d}^{\prime}$ impression et de la censure ${ }^{3}$, contrôle étroit de la presse. Ce système persista dans ses formes premières jusqu'à la Révolution, qui établit une " liberté de la presse ", vite étouffée par les événements politiques de la fin du siècle. Cette conférence a pour but de présenter ce système, ses ambiguïtés, l'art d'en contourner les règles tant dans le domaine de l'imprimé que de la communication manuscrite.

L'histoire de la presse française ancienne a été essentiellement faite depuis trente ans par des dix-huitiémistes, et ce n'est pas dans un congrès dix-huitiémiste qu'il faut s'en plaindre, d'autant que plusieurs collègues canadiens participèrent aux grandes aventures animées par Jean Sgard que furent le Dictionnaire des journalistes (1600-1789) publié en $1976^{4}$ et largement révisé ensuite par cinq Suppléments ${ }^{5}$ avant de paraître sous sa forme définitive (pour un temps) en $1999^{6}$; le Dictionnaire des journaux (1600-1789) l'avait précédé en 19917 , qui multipliait par trois (1267) le nombre des périodiques de langue française catalogués au XIX ${ }^{\mathrm{e}}$ siècle par le bibliographe Eugène Hatin ${ }^{8}$. Nous parlons de langue française, car si ces dictionnaires s'intéressaient à tous les périodiques publiés dans le ressort de la France au sens d'aujourd'hui et de la francophonie (Pays-Bas autrichiens et Suisse), ils recensaient aussi tous ceux qui avaient choisi d'utiliser le medium de la langue de Théophraste Renaudot, le créateur de la Gazette, pour informer, voire pour désinformer leurs contemporains : en Hollande et en Allemagne, en Angleterre et en Italie, mais aussi en Pologne et en Russie, la presse de langue française fut présente à tel ou tel moment du XVIII siècle?

3 Lucien Febvre et Henri-Jean Martin, L'Apparition du livre, postface de Frédéric Barbier, Paris, Le Grand Livre du mois, 1999 (première édition : Paris, Albin Michel, 1958).

4 Presses universitaires de Grenoble (PUG), 1976, 1 vol.

5 Anne-Marie Chouillet et François Moureau (préparé par), Dictionnaire des journalistes, 1600-1789: supplement, Grenoble, Centre de recherche sur les sensibilités, Université des langues et lettres de Grenoble, 1980-1987.

6 Oxford, Voltaire Foundation, 1999, 2 vol.

7 Paris, Universitas, 1992, 2 vol.

8 Bibliographie historique et critique de la presse périodique française, Paris, Firmin Didot, 1866.

9 Liste dans le Dictionnaire des journaux, t. II, p. 1144-1146. 
Dans les îles françaises de la Caraibe, on imprima quatorze journaux ou gazettes ${ }^{10}$, dont une Gazette de la Martinique (1766-1793) ${ }^{11}$ et une Gazette de Sainte-Lucie (1787-1793) ${ }^{12}$ très sensibles à l'air du temps au moment de la Révolution. En Amérique du Nord même, on publia deux périodiques à Québec ${ }^{13}$ et autant à Montréal ${ }^{14}$, et un à Boston, Newport, la Nouvelle-Orléans et Philadelphie ${ }^{15}$, dont les notices pour le dictionnaire furent rédigées par Jean-Paul de Lagrave, Marie-France Silver ou Alain Nabarra. Aucun de ces journaux ou gazettes n'est antérieur au Traité de Paris, qui règle la présence française en Nouvelle-France. On peut alors parler d'une véritable presse "américaine", souvent inspirée au Bas-Canada par les intérêts britanniques. La Gazette française (de Newport) appartient à une catégorie très particulière de journal de nouvelles et, encore plus, à une pratique typographique connue sous le nom d'impression d'escadre ${ }^{16}$. C'est, en effet, grâce à une presse montée sur Le Neptune, un vaisseau du corps expéditionnaire commandé par le comte de Rochambeau, que la gazette parut de novembre 1780 au printemps 1781. Il s'agit moins d'une feuille de propagande, comme l'avait été le Journal militaire ${ }^{17}$ publié à Hanovre en 1757 sur les presses de l'armée du maréchal-duc de Richelieu pour la clientèle locale, que d'un périodique donnant des informations « américaines » en français destinées à l'encadrement militaire français. Ces derniers exemples montrent que la presse a plus d'une fonction, mais qu'elle est toujours

10 Dictionnaire des journaux, $\mathrm{n}^{\circ} 18,28,480,496,509,510,518,523,526,543,620,686$, 702, 1071, plus un Mercure des Antilles (1783) à Kingston en Jamaïque, $\mathrm{n}^{\circ} 933$.

11 Dictionnaire des journaux, $\mathrm{n}^{\circ} 518$.

12 Dictionnaire des journaux, $\mathrm{n}^{\circ} 526$.

13 Dictionnaire des journaux, n²82, 521 : Courrier de Québec (1788) ; Gazette de Québec (1764-1779, 1785-1794).

14 Dictionnaire des journaux, n 519, 556 : Gazette de Montréal (1785-1794) ; Gazette du commerce et littéraire, pour la Ville et District de Montréal (1778-1779).

15 Dictionnaire des journaux, $\mathrm{n}^{\circ}$ 264, 563, 252, 266 : Courrier de Boston (1789); Gazette française (1780-1781) ; Le Courrier (1785-1786 ?); Courrier de l'Amérique (1784).

16 Magali Vène, "Les impressions d'escadre françaises de la fin du XVII ${ }^{\mathrm{e}}$ siècle au XVIII ${ }^{\mathrm{e}}$ siècle", dans Annie Charon, Thierry Claerr et François Moureau (textes réunis par), Le Livre maritime au siècle des Lumières. Édition et diffusion des connaissances maritimes (1750-1850), Paris, Presses de l'Université Paris-Sorbonne, 2005, p. 81-82.

17 Dictionnaire des journaux, $\mathrm{n}^{\circ} 770$. 
très encadrée. Cela nous ramène à la situation de celle-ci dans le royaume de France avant la Révolution.

L'exercice de l'imprimerie y est réputé une activité libérale, par laquelle les maîtres parisiens de la Communauté des Libraires sont " censés et réputés du Corps et des Suppôts de l'Université de Paris, distingués et séparés des Arts mécaniques ", si l'on en croit tous les textes officiels et leur jurisprudence compilés dans le Code de la librairie, dit Saugrain, publié en $1744^{18}$. La législation de la presse est, elle aussi, tributaire du même code, qui ne distingue pas les périodiques des autres types d'impression ${ }^{19}$. Au XVIII ${ }^{\mathrm{e}}$ siècle encore, on désigne sous le nom de "livre » les journaux du type des Mercures dont le format in-12 et le système de censure sont identiques à ceux des ouvrages de littérature. Bien que le nombre des imprimeurs et des presses autorisés ait été graduellement limité par le pouvoir royal sous Louis XIV, le " libraire » est théoriquement à même de mettre sous la presse ce qu'il souhaite, de " tout imprimer librement, sous l'inspection de deux ou trois censeurs », dirait Figaro ${ }^{20}$. Par divers règlements promulgués par Richelieu au nom de Louis XIII (1626-1629) ${ }^{21}$, le système est parfaitement rodé avant même la création de la première gazette en 1631: le libraireimprimeur fournit le manuscrit à un censeur nommé par le Chancelier, qui lit et paraphe l'ouvrage et lui accorde ou non une "approbation " dont le libellé sera reproduit dans le livre. Cette approbation est juge de la conformité du texte avec les idées communément reçues. L'auteur ou, plus souvent, le libraire-imprimeur sollicite ensuite en Conseil du roi un "privilège à temps » qui protège le «droit de copie » du bénéficiaire et interdit - théoriquement - la contrefaçon ${ }^{22}$. Ces privilèges,

18 Claude-Marin Saugrain, Code de la Librairie et Imprimerie de Paris ou Conférence du Règlement arrêté au Conseil d'État du Roi le 28 février 1723, et rendu commun pour tout le Royaume, par arrêt du Conseil d'État du 24 mars 1744, avec les anciennes ordonnances, édits, déclarations, arrêts, règlements et jugements rendus au sujet de la Librairie et de l'Imprimerie, depuis l'an 1332, jusqu'à présent, Paris, Aux dépens de la Communauté, 1744 , Titre I : «Des franchises, exemptions et immunités des imprimeurs et des libraires de Paris », p. 5 (Règlement de 1723 élargi au royaume en 1744).

19 D'où l'absence totale de la presse dans un code d'une précision maniaque sur les aspects les plus variés de la production, de la commercialisation et du contrôle du livre.

20 Beaumarchais, Le Mariage de Figaro, acte V, scène 3.

21 Code de la Librairie et Imprimerie de Paris, Titre XV : « Des privilèges et continuations d'iceux pour l'impression des livres », article CI, p. 358-359.

22 François Moureau (textes réunis par), Les Presses grises. La Contrefaçon du livre (XVI'-XIX siècles), Paris, Aux Amateurs de Livres, 1988. 
pour la plupart de trois ans, sont éventuellement renouvelables par " continuation ». Pour les brochures de deux feuilles au plus (48 p. in12), une simple permission d'imprimer est accordée par le Lieutenant général de police du lieu ${ }^{23}$.

Le statut de la presse périodique n'est pas distinct en droit de celui de l'édition en général, mais il se combine, au XVII ${ }^{\mathrm{e}}$ et encore largement au XVIII ${ }^{\mathrm{e}}$ siècle, avec celui des compagnies ou manufactures royales à monopole du type de celui de la Compagnie des Indes orientales (1664) ou de la Compagnie des Glaces de Saint-Gobain (1665) : un secteur du commerce ou de l'industrie est réservé à une structure financière privée dotée d'un privilège d'exclusivité par le pouvoir royal ${ }^{24}$. Ce système lié au mercantilisme d'État ${ }^{25}$ se mettra en place sous le ministériat de Jean-Baptiste Colbert dans les premières années du règne personnel de Louis XIV. Mais il avait été précédé d'une ébauche avec la création de la Gazette en $1631^{26}$. Auparavant existaient des annales sous la forme du Mercure français dont la publication s'étendit de 1613 à $1648^{27}$; mais la Gazette était une création ex nihilo qui établissait dans le domaine de l'information politique ce que l'on appelait "l'Exclusif» dans le domaine commercial ${ }^{28}$. Un «privilège général » sans durée fixée, mais révocable ad nutum, était accordé à une personne - en l'occurrence le médecin Théophraste Renaudot (1586-1653) ${ }^{29}$ - qui pouvait le transmettre comme un bien propre à ses descendants; ce qui fut le cas ${ }^{30}$. Plus tard, Diderot, très inspiré par la Communauté des Libraires parisiens,

Code de la Librairie et Imprimerie de Paris, Titre XV, article CII, p. 369.

24 Voir les articles «INDUSTRIE » et « MANUFACTURES » dans Lucien Bély (sous la direction de), Dictionnaire de l'Ancien Régime, Paris, PUF, 1996, p. 658-661 et p. 789-790

25 Article « Mercantilisme » dans le Dictionnaire de l'Ancien Régime, op. cit., p. 816817.

26 Dictionnaire des journaux, $\mathrm{n}^{\circ} 492$.

27 Dictionnaire des journaux, $\mathrm{n}^{\circ} 937$

28 Obligation faite aux colonies de ne commercer qu'avec la Métropole : voir l'article « ExCLuSIF » du Dictionnaire de l'Ancien Régime, op. cit., p. 526-527.

29 Dictionnaire des journalistes, $\mathrm{n}^{\circ} 677$.

30 La Gazette rapportait 15000 livres par an à Renaudot dans les années 1640 (BnF, ms., fr. 22804, f. 22). Son fils, Théophraste II, puis ses petit-fils François et Eusèbe Renaudot (1648-1720) (Dictionnaire des journalistes, $\mathrm{n}^{\circ}$ 676) et leur neveu EusèbeJacques Chaspoux de Verneuil (1695-1747) et son fils Eusèbe-Félix bénéficièrent jusqu'en 1749 des revenus du privilège et de la charge de la Gazette. Á cette date, le privilège fut vendu par Chaspoux de Verneuil à Pierre-Nicolas Aunillon (16841758) (Dictionnaire des journalistes, $n^{\circ} 23$ ). Par lettres patentes d'août 1761 , le minis- 
défendra les «privilèges généraux » de librairie comme des biens meubles transmissibles contre la volonté d'un pouvoir royal soucieux d'en limiter l'exercice et les bénéfices parfois exorbitants ${ }^{31}$.

Confronté à une littérature pamphlétaire clandestine, Richelieu considéra dès l'origine la Gazette comme un medium de propagande dont les réimpressions provinciales assuraient la diffusion dans les régions les plus reculées du royaume ${ }^{32}:$ le cardinal-ministre relisait la copie et y contribuait lui-même, imité en cela par le roi $^{33}$. Hebdomadaire de 8 pages, la forme "gazette » fut copiée ensuite par toute sortes de journaux de nouvelles en Europe : classées du plus loin au plus près, des nouvelles étrangères aux nouvelles nationales et de la Cour ${ }^{34}$, les informations donnaient le ton de ce qu'il fallait penser ; quand, en 1762, les Affaires étrangères prirent la direction officielle de la Gazette de France, il fut encore plus évident qu'elle était, si l'on peut dire, la « voix de la France ». Critiquant le style convenu, voire convenable, de ce type de périodique, Voltaire note : «Les Gazettes de France ont toujours été revues par le Ministère ${ }^{35}$ ». Mais l'opinion publique n'était pas dupe et elle se défiait des informations fournies par ce medium très officiel : l'un des personnages d'un conte de fées libertin composé en 1760 par CharlesAlexandre de Calonne, le futur ministre de Louis XVI, témoigne sur le mode satirique du peu de confiance qu'on avait dans les nouvelles: ayant reçu avis du futur mariage de sa fille, le roi des Patagons - antihéros du conte — « fut étonné de la nouvelle : il l'avait pourtant apprise

tère des Affaires étrangères prit en gestion directe la rédaction et la propriété de la Gazette devenue Gazette de France de 1762 à 1792.

31 «Lettre historique et politique adressée à un magistrat sur le commerce de la librairie " (1763), dans CEuvres, t. III: Politique, éd. Laurent Versini, Paris, Robert Laffont, coll. "Bouquins », p. 59-116. Voir le Code de la Librairie et Imprimerie de Paris, p. 366-367 (« Autorités contre des privilèges généraux et d'espèce particulière ", entre 1674 et 1721 ).

32 Gilles Feyel, La Gazette en province à travers ses réimpressions (1631-1752), Amsterdam et Maarssen, APA-Holland University Press, 1982.

33 Gilles Feyel, «Richelieu et la Gazette aux origines de la presse de propagande ", dans Roland Mousnier (sous la direction de), Richelieu et la culture, Paris, Éditions du CNRS, 1987, p. 103-123.

34 Stéphane Haffemayer, «La géographie de l'information dans la Gazette de Renaudot de 1647 à 1663 ", dans Gazettes et information politique sous l'Ancien Régime, Henri Duranton et Pierre Rétat éd., Publications de l'Université de Saint-Étienne, 1999, p. 21-29.

35 «De la gazette", Nouveaux Mélanges philosophiques, historiques, critiques, [Genève, Cramer], 1764, t. II, 335-338 (article de l'Encyclopédie), p. 336. 
par la Gazette ; mais il n'en croyait rien ${ }^{36}$. Ce que disent discrètement les petits romans libertins tolérés, le public des cafés, des cercles bourgeois et des salons aristocratiques le répétait sans qu'on en ait conservé la trace.

Le monopole théorique de la Gazette dura jusqu'à la Révolution, et la création d'autres journaux de nouvelles politiques fut toujours soumise dans le royaume à l'accord rémunéré du propriétaire de la Gazette $e^{37}$. Nouveau magnat de la presse parisienne dans les années 1770, CharlesJoseph Panckoucke ${ }^{38}$ publia avec l'aval du Département des Affaires étrangères deux journaux politiques censés être imprimés à Genève et à Bruxelles ${ }^{39}$, une forme de "permission tacite » appliquée à la presse ${ }^{40}$ qui permettait aussi d'exonérer le pouvoir royal de toute responsabilité dans la rédaction des nouvelles, voire de favoriser telle ou telle désinformation. Mais ce fut surtout la presse d'annonces, dite Annonces, affiches et avis divers, qui, à partir du milieu du XVIII ${ }^{\mathrm{e}}$ siècle, renouvela la physionomie de la presse d'information générale: sous le contrôle discret des intendants, ces périodiques imprimés en province ${ }^{41}$ correspondait en gros à ce qui est aujourd'hui la presse régionale. Les informations de proximité y voisinaient avec ce que l'on pourrait appeler la pédagogie d'une certaine modernité favorisée par la haute administration royale: agronomie, technologie, médecine, etc. Un lectorat nouveau

36 Tant mieux pour elle, conte plaisant. Il y a commencement à tout, s.l.n.d. [Paris, 1760], p. 22. Le conte est aussi attribué à l'abbé de Voisenon.

37 Gilles Feyel, L'Annonce et la nouvelle. La Presse d'information en France sous l'Ancien Régime, Oxford, Voltaire Foundation, 2000.

38 Dictionnaire des journalistes, $\mathrm{n}^{\circ}$ 616; Suzanne Tucoo-Chala, Charles-Joseph Panckoucke et la librairie française, 1736-1798, Pau, Marrimpouey, 1977.

39 Journal historique et politique (1772) et Journal de politique et de littérature (1774) (Dictionnaire des journaux, $\left.\mathrm{n}^{\circ} 754,684\right)$. Voir $\mathrm{G}$. Feyel, L'Annonce et la nouvelle, op. cit., p. 836.

40 Imaginée sous la Régence de Philippe d'Orléans pour protéger le marché français contre l'édition étrangère, la " permission tacite " était une tolérance de publication et de diffusion en France pour des ouvrages imprimés dans le royaume, mais sous fausse adresse étrangère. Il en était tenu registre officiellement, bien que le Code de la librairie n'en dise mot, bien entendu. Voir François Moureau, La Plume et le plomb. Espaces de l'imprimé et du manuscrit au siècle des Lumières, Paris, Presses de l'Université Paris-Sorbonne, coll. « Lettres françaises », 2006, p. 242-244.

41 Jean Sgard (sous la direction de), La Presse provinciale au XVIII siècle, Grenoble, Centre de Recherche sur les Sensibilités, 1983, p. 25-130 (table alphabétique des titres d'Annonces). Les premières paraissent à Lyon en 1750, un an avant celles de Paris, dites Petites Affiches de Paris. 
y puisait quelque chose de l'esprit des Lumières marqué par un fort patriotisme régional ${ }^{42}$. Les responsables des Bureaux d'Adresse provinciaux qui géraient les Annonces étaient des «fermiers » de la direction de la Gazette de France qui leur déléguait par bail le droit d'impression et de diffusion ${ }^{43}$. Un an avant la Révolution, il existait quarante-quatre feuilles de ce type ${ }^{44}$.

Dans une monarchie qui avait organisé, dès le XVII ${ }^{\mathrm{e}}$ siècle, un système académique rapidement chargé de magnifier « le plus grand roi du monde ${ }^{45}$, il était évident qu'aucun des domaines de l'esprit n'était oublié : Académie française (1635), Académie de peinture et de sculpture (1648), Académie des sciences (1666), Petite Académie (1663) chargée de réaliser les devises pour les monuments à la gloire du roi et devenue Académie des Inscriptions (1716), Académie diplomatique (1712), Académie de marine (1752). Ces institutions pensionnées par le pouvoir d'État avaient plusieurs fonctions ${ }^{46} ; l^{\prime}$ une d'entre elles était la diffusion du progrès des sciences et des connaissances en général. Outre des "mémoires » publiés annuellement, elles pouvaient être en charge d'une plus large communication par l'intermédiaire de la presse. Ce fut l'origine du Journal des savants ${ }^{47}$ créé en 1665 selon le principe du privilège personnel à monopole: il existe encore en 2009 sous la forme d'une revue patronnée par l'Académie des Inscriptions... En 1701, l'abbé Jean-Paul Bignon, directeur de la Librairie - une espèce de ministre de la Culture - et surtout neveu du chancelier de Pontchartrain, prit en main le Journal des savants et s'entoura d'un comité de rédaction composé d'académiciens pensionnés pour cette tâche. Cette structure persista jusqu'en 1792, date de la première suppression du

42 Jean Sgard, «La presse provinciale et les Lumières ", dans J. Sgard (sous la direction de), La Presse provinciale..., op. cit., p. 49-64.

43 Gilles Feyel, «La presse provinciale sous l'Ancien Régime », dans Jean Sgard (sous la direction de), La Presse provinciale ..., op. cit., p. 8-9

44 Ibid., p. 17-20.

45 Lucien Bély, Louis XIV. Le plus grand roi du monde, s.l., Jean-Paul Gisserot, 2005.

46 Voir les divers articles sur ces académies dans le Dictionnaire de l'Ancien Régime, op. cit., p. 9-19. On pourrait y ajouter l'Académie de danse (1662) et l'Académie de musique (dite Opéra) (1669-1672) (Marcelle Benoit (sous la direction de), Dictionnaire de la musique en France aux XVII et XVIII siècles, Paris, Fayard, 1992, p. 1-4), plus la Comédie-Française (1680), troupe royale à monopole.

47 Dictionnaire des journaux, $\mathrm{n}^{\circ} 710$; Jean-Pierre Vittu, " La formation d'une institution scientifique : le Journal des savants de 1665 à 1714 ", Journal des savants, 2002, p. 179-204 et p. 349-378. 
périodique. Á l'origine, le Journal des savants avait le monopole sur de vastes domaines, sur " ce qui se passe de nouveau dans la République des Lettres", allant des sciences exactes aux sciences historiques, par l'intermédiaire de "mémoires ", $\mathrm{d}^{\prime}$ " extraits » et de comptes rendus d'ouvrages et d'expériences. Dix volumes de tables analytiques furent publiés de 1753 à 1764 par Antoine Briasson, l'un des quatre libraires de l'Encyclopédie, sur le modèle de celles des Philosophical Transactions de la Royal Society londonienne traduites en français par François de Brémond dès $1739^{48}$. Malgré la création, dans la seconde moitié du XVIII siècle, de nombreux journaux spécialisés - commerce, agriculture, médecine, physique, etc.- qui empiétaient sur le privilège du Journal des savants, le pouvoir royal tenait à maintenir la fiction des domaines réservés. En 1761 encore, Malesherbes, directeur de la librairie et fils du chancelier de Lamoignon - nouveau Bignon acquis aux idées des nouveaux philosophes, mais magistrat avant tout - répondait sèchement au duc de Choiseul, secrétaire d'État aux Affaires étrangères en charge de la Gazette de France qui souhaitait annoncer des ouvrages nouveaux: "Le privilège exclusif du Journal des savants y est contraire ". Cependant Malesherbes organisa un système de "permissions tacites " pour laisser prospérer des journaux dont le domaine était largement laissé en jachère par le Journal des savants : la seule condition était le paiement au périodique privilégié d'une redevance annuelle fixée en fonction du tirage. Ces pratiques sont tout à fait typiques de la jurisprudence juridique de l'Ancien Régime finissant : les lois et règlements les plus contraignants étaient adaptés par l'administration royale elle-même sous forme de décisions parallèles de simple police, certes "registrées » mais officiellement nulles et non avenues. Dès le début du siècle (1701), les jésuites parisiens du Collège Louis-le-Grand avaient trouvé la parade en faisant paraître à Trévoux, dans la principauté de Dombes réputée terre étrangère, sur les bords de Saône à quelques dizaines de kilomètres au nord de Lyon, des Mémoires pour l'histoire des sciences et des beaux-arts dits Mémoires de Trévoux ${ }^{50}$, "dédiés à Son Altesse Sérénissime Monseigneur le prince souverain de Dombes ", Louis-Auguste de Bourbon, duc du Maine, fils légitimé de Louis XIV et de Mme de Montespan. Ce mensuel savant, auquel participèrent quelques-uns des meilLondres. Depuis 1665 jusqu'en 1735, Paris, Piget, 1739, un vol. in-4². 
leurs esprits du siècle - dont Leibniz ${ }^{51}$ - , quoi qu'en ait dit Voltaire ${ }^{52}$, survécut jusqu'en 1767, au-delà même de la suppression en France de la Compagnie de Jésus (1762). L'imprimerie de Son Altesse Sérénissime servit à beaucoup de choses, dont à l'impression en 1765 des volumes interdits de l'Encyclopédie ${ }^{53}$.

Mais la France qui lisait n'était pas uniquement composée d'amateurs de nouvelles politiques et de comptes rendus savants. La sociabilité mondaine avait une large place dans le système mis en place pour donner à l'extérieur une image d'une France où les arts et les lettres renouvelaient dans le siècle de Louis XIV les siècles de Périclès et d'Auguste. Ce fut, on le sait, la consigne donnée par Charles Perrault, homme de Colbert à l'Académie française, dans son célèbre poèmeprogramme de 1687: moderne, chrétien, c'est-à- dire catholique, monarchique, le Siècle de Louis le Grand ${ }^{54}$ exprimait par les vers du futur auteur des Contes un projet dans lequel la presse avait sa part. Colbert était mort en 1683 et Perrault avait perdu avec lui l'homme de cette politique : la Querelle des Anciens et des Modernes témoigna aussi de cette réalité et des oppositions qui furent aussi politiques ${ }^{55}$. Dès les premières années du règne personnel, Colbert avait formé le projet de créer ce qui fut le premier périodique de simple littérature : on lui donna en 1672 le nom de Mercure galant ${ }^{56}$. Le terme de "galant " n'était pas là par hasard: il signifiait « honnête, civil, sociable, de bonne compagnie, de conversation agréable ${ }^{57}$ "; «qui à l'air de la Cour »,

51 Carlos Sommervogel, Table méthodique des Mémoires de Trévoux (1701-1775), Paris, Auguste Durand, 1864, p. XXII.

52 Surtout contre le père Guillaume-François Berthier, directeur des Mémoires de 1745 à 1762 (Dictionnaire des journalistes, $n^{\circ}$ 68) ; John Pappas, Berthier's Journal de Trévoux and the Philosophes, Genève, Studies on Voltaire and the Eighteenth Century, 3, 1957.

53 François Moureau, Le Roman vrai de l'Encyclopédie, $2^{e}$ éd., Paris, Gallimard, coll. «Découvertes », 2001, p. 110.

54 Paris, Jean-Baptiste Coignard, 1687, 27 p., in $-4^{\circ}$.

55 Voir Anne-Marie Lecoq (édition établie et annotée par, précédée de "Les abeilles et les araignées » par Marc Fumaroli), La Querelle des Anciens et des Modernes, Paris, Gallimard, coll. « Folio », 2001.

56 Dictionnaire des journaux, $\mathrm{n}^{\circ} 919$ (années 1672-1710).

57 Gaston Cayrou, Dictionnaire du français classique. La langue du XVII siècle, Paris, Le Livre de Poche-Klincksieck, 2000 (reprise de la $2^{\mathrm{e}}$ édition de 1924), p. 387. 
précisait même Furetière ${ }^{58}$. D'ailleurs, à l'origine, le Mercure galant se présentait sous la forme d'une lettre mensuelle adressée à une dame de province que lui envoyait celui qui se désignait comme "l'auteur du Mercure ». Cette fiction, reprise des Lettres en vers ${ }^{59}$ publiées dans les années précédentes par Loret et ses successeurs ${ }^{60}$, fut l'œuvre de Jean Donneau de Visé ${ }^{61}$.

Le Mercure galant "contenant plusieurs histoires véritables et tout ce qui s'est passé depuis le $1^{\mathrm{er}}$ janvier 1672 jusques au départ du roi » parut en mai $^{62} ; l^{\prime}$ Avis au lecteur précisait que le périodique «n'a rien qui ressemble au Journal des savants », utile précaution qui délimitait le domaine de compétence de la nouvelle feuille. Très lié dans les années 1660 au milieu précieux, Donneau de Visé sut mêler à l'hagiographie du monarque les échos en prose et en vers de la vie littéraire et mondaine. Il se fit le défenseur des «modernes » en s'attachant la collaboration de leurs représentants les plus éminents, Thomas Corneille et Fontenelle. Ce fut l'image du périodique jusque dans les années 20 du siècle suivant, quand Marivaux y débuta par des «lettres au Mercure » (1717$1720)^{63}$. En 1724, le journal prit le titre de Mercure de France, « dédié au roi ${ }^{64}$ - signe de son allégeance renouvelée. Á travers divers avatars, ce périodique a duré jusqu'en $1965^{65}$; sa maison d'édition existe encore dans le groupe Gallimard. "Moderne » et ouvert aux arts les plus

58 Dictionnaire universel, La Haye et Rotterdam, Arnout et Reinier Leers, 1690, t. I, s.v. "GALANT».

59 La Gazette du temps [à Mlle de Longueville], par Jean Loret (1652) (Dictionnaire des journaux, ${ }^{\circ} 557$; Dictionnaire des journalistes, $\left.\mathrm{n}^{\circ} 528\right)$; La Muse historique (16581659) (Dictionnaire des journaux, $\mathrm{n}^{\circ} 966$ ); Lettres en vers à S.A. Madame la duchesse de Nemours (1659-1671) (Dictionnaire des journaux, $\left.\mathrm{n}^{\circ} 817\right)$, ; Lettres en vers à Madame (1665-1672) (Dictionnaire des journaux, $\left.\mathrm{n}^{\circ} 815\right)$; etc.

60 James de Rothschild (recueilli et publié par), Les Continuateurs de Loret. Lettres en vers de La Gravette de Mayolas, Robinet, Boursault, Perdou de Subligny, Laurent et autres. 1665-1689, Paris, D. Morgand et C. Fatout, 1881-1899, 3 vol.

61 Dictionnaire des journalistes, $\mathrm{n}^{\circ} 244$.

62 Donneau de Visé fait allusion à la « conquête de la Hollande » par les armées françaises. Voir Charles Hénault, Nouvel Abrégé chronologique de l'Histoire de France, Paris, Prault père, 1744, p. 349-351.

63 Dictionnaire des journalistes, $\mathrm{n}^{\circ} 549$.

64 Dictionnaire des journaux, $\mathrm{n}^{\circ} 924$ (années 1724-1778).

65 Voir notre notice « MERCuRE DE FRANCE», dans Jean-Pierre de Beaumarchais, Daniel Couty et Alain Rey (éditeurs), Dictionnaire des littératures de langue française, Paris, Bordas, 1984, t. II, p. 1472-1473. 
divers sous les successeurs de Donneau de Visé - Charles Dufresny $(1710-1714)^{66}$ ou Antoine de la Roque (1724-1744) ${ }^{67}, 1^{\prime}$ ami le plus intime de Watteau ${ }^{68}$-, il devint ensuite " voltairien » ; et Voltaire lui-même lui donna son orientation avec des "Conseils à un journaliste", confiés au premier numéro (novembre 1744) du nouveau privilégié, Charles Le Clerc de la Bruère (1744-1754) ${ }^{69}$. Le terme de «journaliste " semble avoir été inventé par Pierre Bayle en 1684 pour désigner les auteurs de comptes rendus savants ${ }^{70}$. Voltaire voit essentiellement dans le «journalisme » littéraire ${ }^{71}$ une presse de comptes rendus ou d' « extraits », ce qui n'était que pour partie la pratique du Mercure de France, dont les " pièces fugitives » originales en vers et en prose formaient une section développée dès la direction de Dufresny.

Avec les responsables éditoriaux suivants, de l'abbé GuillaumeThomas Raynal (1750-1755) ${ }^{72}$ à Jean-François Marmontel (1755-1758) ${ }^{73}$, le voltairianisme du Mercure s'accentua encore; il consistait en un esprit des Lumières adapté aux réalités politiques et sociales du temps qui prenait corps dans des institutions comme l'Académie-Française ou la Comédie-Française. Donneau de Visé avait fait une fortune

66 Dictionnaire des journaux, $\mathrm{n}^{\circ} 920$; François Moureau, Le Mercure galant de Dufresny (1710-1714) ou le Journalisme à la mode, Oxford, Studies on Voltaire and the Eighteenth Century 206, 1982.

67 Dictionnaire des journalistes, $\mathrm{n}^{\circ} 459$.

68 François Moureau, «De Watteau à Chardin : Antoine de la Roque, journaliste et collectionneur ", dans Mélanges en hommage à Pierre Rosenberg: Peintures et dessins en France et en Italie, XVII $-X V I I I^{e}$ siècles, Paris, Réunion des Musées nationaux, 2001, p. 349-355.

69 Dictionnaire des journaux, $\mathrm{n}^{\circ} 483$. Voir notre édition des « Conseils » dans les CEuvres complètes de Voltaire, Oxford, Voltaire Foundation, 2003, t. 20A : «CEuvres de 1739$1741 »$, p. 449-516.

70 Préface à la première livraison des Nouvelles de la République des Lettres, Amsterdam, Henry Desbordes, mars 1684, n. p. : "Car il y a des ouvrages très curieux, qui viennent en même temps à la connaissance de plusieurs pays étrangers, et alors chaque journaliste se hâte d'en faire mention " (c'est nous qui soulignons). Le Dictionnaire universel (1690) de Furetière, qui répertorie "gazette » et " gazetier », ignore « journaliste », qui est présent dans la dernière édition du Dictionnaire universel français et latin dit de Trévoux (Paris, Libraires associés, 1771, t. V).

71 Le terme de « journaliste " s'oppose à celui de " gazetier », comme celui de « journal » (périodique de comptes rendus) à celui de " gazette » (périodique d'informations générales et politiques).

72 Dictionnaire des journalistes, $\mathrm{n}^{\circ} 666$.

73 Dictionnaire des journalistes, $\mathrm{n}^{\circ} 550$. 
considérable; ensuite, des pensions furent attachées au "brevet de composition du Mercure » délivré en Conseil du roi ; elles récompensaient des écrivains méritants, qui pouvaient perdre cet avantage si le privilégié était démis ${ }^{74}$. Cette habile manière de contrôler le directeur du périodique par les hommes de lettres eux-mêmes se combinait avec l'intervention du pouvoir dans les secteurs sensibles comme l'information internationale ou la Cour, qui étaient confiées en sousmain à des responsables dont on a la liste dès la Régence ${ }^{75}$. C'est alors, dans les années 1720, où l'on oublie la rigueur louis-quatorzienne, que se multiplient des périodiques devant allégeance financière au Mercure : il s'agit souvent de journaux à rédacteur unique sur le modèle du Mercure galant primitif; on les appelle des "spectateurs" à l'imitation du Spectator anglais (1711-1712) de Richard Steele et Joseph Addison qui en est le modèle reconnu. Le Spectateur français $(1721-1724)^{76}$ de Marivaux reste aujourd'hui la plus célèbre de ces feuilles où s'illustrèrent aussi quelques journalistes femmes ${ }^{77}$ bénéficiant de cette forme assez libre qui associait l'intime au général. Marivaux élargit encore cette formule avec des journaux moraux dont les plus originaux sont Le Cabinet $d u$ philosophe (1726) et L'Indigent philosophe (1734). Ils sont les fils ou frères plus ou moins légitimes des petites feuilles «à six sols", brochures périodiques à coloration morale publiées avec permission simple donc sans véritable censure - depuis les premières années du siècle.

$\mathrm{Au}$ fil des décennies du très long règne de Louis XV (1715-1774), le Mercure céda de larges parts de marché à divers journaux dont les secteurs d'information étaient pourtant de son ressort exclusif. Ce furent, entre autres, les périodiques de Pierre François Guyot Desfontaines $^{78}$ ou d'Élie Fréron ${ }^{19}$ - le « Frélon » ou « Guasp » de Voltaire dans la

74 En 1754, les pensions sur le Mercure s'élèvent à $12400 £$ selon Charles Collé (Journaux et Mémoires, Paris, Didot, 1868, t. I, p. 437) : $2000 £$ pour Raynal et Marmontel, $1200 £$ à Piron, etc.

75 Dictionnaire des journaux, $\mathrm{n}^{\circ} 923$ (Le Mercure, années 1721-1723). Liste aux AnF, $\mathrm{AJ}^{13}$ 1034-13 dans les papiers de Louis Fuzelier, co-privilégié du Mercure (Dictionnaire des journalistes, $\mathrm{n}^{\circ} 321$ ).

76 Dictionnaire des journaux, $\mathrm{n}^{\circ} 1217$. Alexis Lévrier, Les Journaux de Marivaux et le monde des 'spectateurs', Paris, Presses de 1'Université Paris-Sorbonne, 2007.

77 Suzanna Van Dijk, Traces de femmes. Présence féminine dans le journalisme français du XVIII siècle, Amsterdam et Maarsen, APA-Holland University Press, 1988. (Liste des journaux publiés au XVIII siècle par des femmes, p. 292-293.)

78 Dictionnaire des journaux, $\mathrm{n}^{\circ} 383$.

79 Dictionnaire des journaux, $\mathrm{n}^{\circ} 316$. 
comédie satirique de $L^{\prime}$ Écossaise ${ }^{80}$-, qui menèrent contre les Philosophes sinon contre les Lumières des combats acharnés plus ou moins protégés par le pouvoir, qui s'irritait souvent de ces querelles considérées comme contraires à la paix civile ${ }^{81}$.

En 1778, Panckoucke obtint des Affaires étrangères et du ministre Vergennes « le privilège exclusif et les brevets des journaux politiques », ce qui faisait du magnat de la presse parisienne le propriétaire du Mercure de France ${ }^{82}$ et l'autorisait, comme nous l'avons dit pour les "gazettes", à multiplier les «journaux» les plus divers, terme équivoque qui désignait tout ce qui n'avait pas la périodicité bi-hebdomadaire des gazettes. La fiction des trois secteurs de l'information devenait de plus en plus difficile à tenir.

Dès l'année précédente - 1777 - , avait été créé le premier quotidien, un Journal de Paris $^{83}$, qui était aussi la première feuille ressemblant à ce que nous nommons aujourd'hui un « journal » d'information générale : nouvelles politiques, actualité littéraire et des théâtres, mode, météorologie, etc. Ce journal était l'œuvre en sous-main du directeur général des finances, le Genevois Jacques Necker. Parmi ses responsables, on trouvait des coreligionnaires de la République de Genève et des banquiers de même origine. La nouvelle politique réformatrice du remplaçant de Turgot au contrôle général des Finances y était évidemment encensée. Le Journal de Paris survécut au renvoi de Necker en 1781 . On voit que les convulsions qui vont mener à la Révolution donnent de plus en plus d'importance à la presse en tant que véhicule de l'opinion publique ou en tant que régulatrice de celle-ci ${ }^{84}$.

Car la réalité lisse de la presse officielle cache une situation largement plus contrastée. Comme l'écrivait rétrospectivement Pierre Manuel sous la Révolution: "Un peuple qui veut s'instruire ne se contente pas de la Gazette de France »" L'illusion colbertiste du "pré

80 F. Moureau, La Plume et le plomb, op. cit., p. 288-289, 374-375.

81 Voir la synthèse de Jack Censer, The French Press in the Age of Enlightenment, London and New York, Routledge, 1994 : "The French Government and the Periodical", p. 138-183.

82 Dictionnaire des journaux, $n^{\circ} 925$ (années 1778-1791).

83 Dictionnaire des journaux, ${ }^{\circ} 682$ (années 1777-1840).

84 J. A. W. Gunn, Queen of the World: Opinion in the Public Life of France from the Renaissance to the Revolution, Studies on Voltaire and the Eighteenth Century 328, 1995.

85 La Police de Paris dévoilée, Paris, J.-B. Garnery, An 2 [1793], t. I, p. 201. 
carré ", d'une France totalement contrôlée de l'intérieur, ne pouvait résister à plusieurs facteurs qui se développèrent au cours du dernier siècle de l'Ancien Régime : les circuits clandestins du livre qui partaient des divers Refuges où l'on retrouvait les huguenots français et les malpensants en général, entre Hollande, Grande-Bretagne, Suisse, voire Allemagne ; la perméabilité du marché français tolérée par le pouvoir royal qui savait faire d'un certain laisser-aller une soupape de sureté conforme à l'esprit nouveau qui avait envahi les plus hautes sphères de l'État-Malesherbes, directeur de la librairie, en fut à la fois l'exemple et la victime ${ }^{86}$; la prise de conscience par la monarchie française que la presse étrangère censément libre était plus à même de gérer l'opinion publique dans la mesure où on pouvait avoir prise sur elle en favorisant ou en interdisant sa diffusion en France.

Á la fin des années 1670, la Ferme générale des Postes, chargée de l'importation des gazettes étrangères, s'était arrangée avec un libraire parisien pour vendre ou louer à un tarif prohibitif ces feuilles assez rares. Mais cela ne favorisait pas la politique de diffusion contrôlée qui restait l'objectif du pouvoir. La vraie réforme se fit avec le "Bureau général des Gazettes étrangères " créé en 1759. De façon très politique, «le privilège exclusif de commerce » de ces feuilles fut accordé conjointement à un protégé du ministre Choiseul, Charles Palissot - qui, l'année suivante, fit jouer la comédie des Philosophes, brûlot des anti-Lumières -, et à Michel-Antoine David, l'un des libraires de l'Encyclopédie. En 1767, les Affaires étrangères, déjà en possession de la Gazette de France, prirent en main directement le Bureau ${ }^{87}$. C'est ainsi que le lectorat de ces gazettes fut élargi par la réduction du coût des abonnements et des feuilles au numéro, et, en partie aussi, par les abonnements partagés, par la location des feuilles chez des " gazetiers » et par le développement des cabinets de lecture parisiens ou provinciaux ${ }^{88}$. Cette évolution mit un terme à la contrefaçon des gazettes étrangères qui était l'un des

86 Pierre Grosclaude, Malesherbes témoin et interprète de son temps, Paris, Fischbacher, 1961, et Malesherbes et son temps (Suite). Nouveaux documents inédits, Paris, Fischbacher 1964.

87 Gilles Feyel, "La diffusion des gazettes étrangères en France et la révolution postale des années 1750 », dans Henri Duranton, Claude Labrosse et Pierre Rétat (textes réunis par), Les Gazettes européennes de langue française (XVII -XVIII siècles), Publications de l'Université de Saint-Étienne, 1992, p. 81-98.

88 Paul Benhamou, «Inventaire des instruments de lecture publique des gazettes », dans H. Duranton et al. (textes réunis par), Les Gazettes européennes ..., op. cit., p. 121-129. 
exercices habituels d'une librairie provinciale en crise face à des libraires parisiens aux ambitions insatiables.

L'imprimerie étant une activité de main-d'œuvre, bruyante, demandant des surfaces importantes pour ses diverses étapes industrielles (depuis la composition typographique jusqu'au brochage du produit fini), localisée dans des secteurs bien délimités des villes, comme la rue Saint-Jacques à Paris et le Quartier latin, on peut s'étonner que la police du livre ait montré une telle inaptitude à contrôler ce qui sortait de ces ateliers. Outre les ouvrages de "permission tacite " déjà signalés, la production imprimée illicite était le lot quotidien des imprimeurs, fussent-ils imprimeurs et "suppôts » officiels du Clergé ou du Parlement ${ }^{89}$ ! Diffuser régulièrement les livraisons $d^{\prime}$ un périodique sans autorisation était une autre affaire. C'est pourquoi un seul réussit à le faire, et ce sur plus de soixante ans : les Nouvelles ecclésiastiques (17281803) ${ }^{90}$ jansénistes furent organisées de telle sorte - et elles en firent même la publicité ${ }^{91}$ - que la communication entre les divers acteurs (rédacteurs, imprimeurs, colporteurs) était automatiquement rompue dès que la police s'était emparée d'un de ces coopérateurs.

Mais ces mêmes Nouvelles ecclésiastiques avaient été précédées, pendant près de vingt ans (1675-1693), d'une forme manuscrite, que l'on nomme génériquement des «nouvelles à la main ». Nous avons publié un vaste répertoire de cette presse manuscrite clandestine de langue française qui va du XVI ${ }^{\mathrm{e}}$ siècle (1532) aux premières années de la Révolution, où cette forme d'information disparut ${ }^{92}$. Mgr Louis Foucquet, évêque $d^{\prime}$ Agde et frère de l'ancien surintendant des Finances abattu par Colbert, avait organisé des réseaux d'une redoutable efficacité, multipliant les correspondants et les ateliers de copie pour servir la pro-

89 Par exemple, la Lettre sur les aveugles de Diderot fut imprimée en 1749 sous la fausse adresse de «Londres" par Claude Simon, « imprimeur de Monseigneur l'archevêque de Paris" (David Adams, Bibliographie des œuvres de Denis Diderot, Ferney-Voltaire, Centre international d'étude du XVIII ${ }^{e}$ siècle, 2000, t. II, p. 216 ; Jean-Dominique Mellot et Élisabeth Queval, Répertoire d'imprimeurs/libraires (vers 1500-vers 1810), Paris, Bibliothèque nationale de France, 2004, $\mathrm{n}^{\circ} 4561$, p. 505). L'auteur fut incarcéré au château de Vincennes pendant quatre mois.

90 Dictionnaire des journauxx, $\mathrm{n}^{\circ} 1027$

91 Planche dépliante en frontispice du recueil de 1728-1730 : « Idée de l'ordre observé pour la distribution des Nouvelles ecclésiastiques », reproduit dans F. Moureau, La Plume et le plomb, op. cit., p. 36, fig. 8.

92 François Moureau, Répertoire des nouvelles à la main. Dictionnaire de la presse manuscrite clandestine. XVI ${ }^{\mathrm{e}}$-XVIII siècle, Oxford, Voltaire Foundation, 1999. 
pagande des "Amis de la Vérité " ${ }^{93}$, ainsi que se désignaient les jansénistes. Mouvement autant politique que religieux au XVIII siècle, très présent dans le milieu parlementaire, le jansénisme reprenait en fait une ancienne pratique de nouvelles manuscrites née vraisemblablement à Rome dans les milieux de la diplomatie vaticane, seule organisation internationale du temps ayant des ramifications sur divers continents, Asie et Amérique comprises ${ }^{94}$. Au cours des guerres de Religion, le futur Henri IV avait compris l'intérêt de ces gazettes manuscrites pour soutenir sa cause ${ }^{95}$. Au siècle suivant, le chancelier Séguier et le cardinal Mazarin doublent les courriers diplomatiques par des abonnements à des nouvelles à la main surtout italiennes ${ }^{96}$. Mais la diffusion manuscrite professionnelle de l'information se développa surtout au XVIII ${ }^{e}$ siècle.

Dans notre $\mathrm{XXI}^{\mathrm{e}}$ siècle, l'essentiel des informations est fournie aux périodiques par quelques agences de presse internationales. On peut se demander comment les gazettes imprimées d'Ancien Régime se procuraient ce qui était leur matière première. Elles pouvaient se copier mutuellement : elles ne s'en privaient pas, même si l'information en perdait de sa fraîcheur. Les sources manuscrites étaient les plus nombreuses. Elles étaient de deux types: l'information internationale était fournie par les rapports diplomatiques réguliers envoyés des postes à Versailles, pour ce qui était du réseau français. Cela explique l'importance des Affaires étrangères dans la gestion de la Gazette de France. Cette information, souvent croisée avec celle de plusieurs postes à l'étranger ${ }^{97}$, était revue par les bureaux de Versailles avant d'être communiquée à la Gazette. Durant les conflits internationaux, le secrétariat d'État à la Guerre ${ }^{98}$ fournissait les fameuses "lettres des armées ", plus ou moins fictives, des espèces de reportages sur le terrain signées d'officiers en opérations que gazettes et mercures $\mathrm{s}^{\prime}$ empressaient de publier ${ }^{99}$. Le secrétariat d'État de Paris et de la Mai-

Répertoire des nouvelles à la main, « Préface », p. XXII, et 1675 (notice 1675.1).

94 Répertoire des nouvelles à la main, " Préface », p. IX. L'inventaire reste encore à faire.

95 Répertoire des nouvelles à la main, 1595 (notice 1595.1).

96 Répertoire des nouvelles à la main, "Préface », p. X.

97 Répertoire des nouvelles à la main, «Préface », p. XV.

98 Art. « SeCRÉTAIRE d’ETAT DE LA GuerRe », dans L. Bély (sous la direction de), Dictionnaire de l'Ancien Régime, op. cit., 1133-1135.

99 Répertoire des nouvelles à la main, "Préface», p. XXXIV; voir, par exemple, 1741(notice 1741.6). 
son du roi ${ }^{100}$ orientait les nouvelles nationales. Mais les gazettes manuscrites privées étaient les plus nombreuses et elles acquirent, surtout à partir des années 1730, une véritable maturité.

Rédigées par des "nouvellistes »-collecteurs, reproduites par une armée de discrets copistes qui donnaient à chaque « ordinaire » la forme canonique des gazettes (classement des nouvelles, numérotation des livraisons, format in $-8^{\circ}$ sur 4 ou 8 pages dont l'adresse), envoyées par voie postale ou diffusées par colportage, les feuilles manuscrites bénéficiaient d'un tirage qui pouvait aller jusqu'à plusieurs centaines de copies et qui n'était pas indigne de celui de nombreuses gazettes imprimées. Des adaptateurs ou de simples contrefacteurs multipliaient encore un lectorat qui pratiquait aussi les abonnements partagés. Chaque « ordinaire ", dans son état originel ou dans ses avatars manuscrits, pouvait atteindre plusieurs milliers de lecteurs. Les séries les plus actives furent, depuis 1740, l'émanation de la "Paroisse Doublet » ${ }^{101}$ à Paris - source manuscrite des Mémoires secrets dits de Bachaumont compilés de 1777 à $1789^{102}$ - ou de Louis-François Mettra ${ }^{103}$, gazetier français installé en Allemagne où il diffusa les « bulletins de Versailles » à partir de $1774^{104}$ source de la Correspondance secrète imprimée (1787-1790 $)^{105}$-, et elles eurent une influence considérable sur l'évolution de l'opinion publique dans les dernières décennies de l'Ancien Régime ${ }^{106}$. Le pouvoir d'État en était parfaitement conscient. Un certain flou juridique entourait ce type de production qui pouvait être considéré, à la limite, comme une simple correspondance personnelle dont l'ordinaire utilisait parfois la forme épistolaire canonique ; on ne pouvait pas, non plus, appliquer la législation du livre à ce qui n'était pas imprimé. Le seul délit punissable en droit était la diffusion de secrets d'État, ce que l'on appelait pompeusement parfois le "secret du Cabinet des Princes ». Au début des années 1740, lors de la Guerre de Succession d'Autriche, on pourchassa

100 Art. « Secrétaire d'Etat de la Maison du RoI », dans Dictionnaire de l'Ancien Régime, op. cit., p. 1135-1140.

101 Sur Marie-Anne Legendre, veuve Doublet, parente de Choiseul, et son milieu proparlementaire - la « Paroisse » - , voir Frantz Funck- Brentano, Figaro et ses devanciers, Paris, Hachette, 1909, p. 257-299.

102 Dictionnaire des journaux, $\mathrm{n}^{\circ} 904$.

103 Dictionnaire des journalistes, $\mathrm{n}^{\circ} 372$.

104 Répertoire des nouvelles à la main, 1774 (notice 1774.7).

105 Dictionnaire des journaux, $\mathrm{n}^{\circ} 235$ (pour la compilation)

106 La Plume et le plomb, op. cit., p. 477-490. 
à Paris les nouvellistes dits « lorrains » soupçonnés de travailler pour la future impératrice Marie-Thérèse et son époux, François de Lorraine ${ }^{107}$. Lors de la Guerre d'Indépendance américaine, on agit de même avec les nouvellistes convaincus d'informer l'Angleterre ${ }^{108}$.

L'administration royale multipliait les interdictions concernant les nouvelles à la main, ce qui prouve le peu d'efficacité de ces arrêts et ordonnances : on en compta cinq de 1656 à 1732, outre un arrêt du Parlement de Paris en $1745^{109}$. Plus habilement, on vit se développer une littérature pamphlétaire contre les «gazetiers » ou "feuillistes » accusés de diffuser de fausses nouvelles et d'inventer le reste: déconsidérer n'était pas une mauvaise méthode ${ }^{110}$. Mais «le Magistrat »-comme l'on disait alors pour désigner et le Lieutenant général de police - comprit rapidement que le système des "gazetins " manuscrits, aussi diversifié qu'impossible à démanteler, pouvait servir de soupape de sureté à une opinion publique assez sceptique sur les nouvelles fournies par les gazettes imprimées. Dès les années 1730, les nouvellistes parisiens les plus en vue collaborèrent étroitement avec le Magistrat ; on en vint à parler de nouvelles "autorisées » et certains gazetiers sollicitèrent des espèces de "privilèges" pour se protéger des contrefacteurs de nouvelles à la main ${ }^{111}$ ! Contre la faculté de servir à quelques riches amateurs des "feuilles particulières » sur les scandales de la vie parisienne - ce qui ne gênait nullement la police - , le Magistrat alimentait la production nouvelliste de «feuilles journalières " concernant le domaine politique que l'on envoyait à un plus large public d'abonnés et auxquelles on joignait parfois des «nouvelles à l'épingle » un peu plus originales ${ }^{112}$ : cet échange de bons procédés satisfaisait les deux parties. Encore fallait-il que cet accord restât secret. Il le fut, sauf pour les autres nouvellistes qui revendiquèrent souvent l'exclusivité curieux écho de la pratique gazetière imprimée pour ces « nouvelles timbrées " ${ }^{113}$ !

107 Voir les Archives de la Bastille (Arsenal, ms. 11544) et Répertoire des nouvelles à la main, 1742 (notice 1742.7).

108 Répertoire des nouvelles à la main, « Préface ", p. XXIII.

109 Ibid., p. XX, n. 75.

110 Ibid., p. XX-XXII.

111 Ibid., p. XXIV.

112 Répertoire des nouvelles à la main, 1740 (notice 1740.4). "Préface », p. XXXVI.

113 Répertoire des nouvelles à la main, 1743 (notice 1743.3). Correspondant d'affaires de Voltaire à Paris et chef de la claque à la Comédie-Française, le chevalier Charles 
Car le monde de la presse connaissait parfaitement l'univers assez bigarré des nouvellistes à la main. Outre des abonnements, qui permettaient de nourrir les feuilles imprimées, à une époque où les agences de presse n'existaient pas ${ }^{114}$, les journalistes virent rapidement dans les feuilles manuscrites un complément intéressant à leur propre activité. Les rédacteurs en charge de périodiques privilégiés organisèrent discrètement des abonnements à des séries de nouvelles à la main, que l'on trouve assez souvent mêlées aux imprimées dans les collections de gazettes $^{115}$. Cela explique pourquoi des privilégiés ou des responsables de la Gazette [de France] comme les abbés Eusèbe Renaudot et Jean-Louis Aubert ou François Marin, des privilégiés du Mercure comme l'abbé François Buchet sont connus pour cette activité, qui dut intéresser plus $\mathrm{d}^{\prime}$ un autre journaliste ${ }^{116}$. Ce revenant-bon financier concernait aussi les gazetiers étrangers de langue française, dont certains comme James de la Cour ${ }^{117}$ à Francfort diffusaient en traduction de très réputées nouvelles à la main britanniques dites "papiers anglais ". Ce moyen de diffusion qui bénéficiait de l'aura particulière du manuscrit illusion du clandestin, donc véridique par rapport à l'imprimé légal était, on vient de le voir, largement surestimé ${ }^{118}$, mais il possédait une vertu supplémentaire : on pouvait en démentir le contenu transmis par des mains anonymes.

Ce fut un exercice que pratiqua une nébuleuse nouvelliste que nous avons appelée l'Agence de Ferney ${ }^{119}$. Avant même son installation à Ferney, dès ses années de Cirey chez Mme du Châtelet, Voltaire, éloigné

de Mouhy obtint la «moitié du privilège des nouvelles» (Répertoire des nouvelles à la main, "Préface», p. XXIV er 1742, notice 1742.4: "Minutes adressées par les ordres du roi et rendues par M. de Marville [Lieutenant de police] au chevalier de Mouhy aux appointements de 1200 livres par an »).

114 F. Moureau, La Plume et le plomb, op. cit., p. 44459-475: «Bureaux de nouvelles et gazettes imprimées ».

115 Par exemple, dans la collection de Philippe-Valentin Bertin du Rocheret à la BnF et à la bibliothèque municipale d'Épernay (Répertoire des nouvelles à la main, "Préface ", p. XIV, n. 27. Corriger les dates : de 1696 à 1738 pour Paris et de 1721 à 1744 pour Épernay).

116 Répertoire des nouvelles à la main, "Préface », p. XXV.

117 Dictionnaire des journaux, $\mathrm{n}^{\circ} 440$. Répertoire des nouvelles à la main, "Préface", p. XXVI.

118 Nous avons fait l'analyse de cette illusion dans La Plume et le plomb, op.cit., p. 1123.

119 La Plume et le plomb, op.cit., p. 445-458: "L'Agence Voltaire de Ferney et d'ailleurs ". 
de la vie littéraire parisienne, comprit que les idées avaient besoin d'un autre medium que le simple imprimé. Voltaire sait se faire interdire à bon escient pour organiser la publicité de telle ou telle production de son esprit; il sait aussi créer autour de lui une attente qui concerne ses faits et gestes : le "roi Voltaire" est le premier grand communicateur de la modernité. Sinon comment expliquerait-on que sa correspondance privée, ses œuvres en vers encore inédites, des anecdotes très personnelles alimentent les nouvelles à la main et font de Voltaire une vedette internationale, dont la réputation va largement au-delà de ceux qui l'ont lu ? Des nouvelles à la main, l'information passe dans les gazettes et les journaux. Voltaire, industriel, sait de même faire la publicité de ses produits de luxe : la duchesse de Choiseul, femme du principal ministre, reçoit les premiers bas de soie de sa manufacture de Ferney, et il le fait savoir. Il fit de même, à partir de 1770, avec sa manufacture de montres, idéalement située aux portes de Genève, dont il offrit un exemplaire orné de diamants à Mme du Barry, la dernière des maitresses en titre de Louis XV. On retrouve cette manière $d^{\prime}$ utiliser le medium manuscrit dans les comptes rendus de Salons diffusés par la Correspondance littéraire de Grimm. Cette feuille bimensuelle manuscrite était envoyée à travers l'Europe à une quinzaine de monarques et de princes qui payaient cher le snobisme d'être informé de ce qui se passait de nouveau à Paris ${ }^{120}$. Tous les deux ans, un salon de peinture, de sculpture et de gravure était organisé au Louvre ; on y voyait l'essentiel de la production artistique ${ }^{121}$. Melchior Grimm eut l'idée de charger Diderot d'en faire la présentation à ses abonnés princiers, parmi lesquels la tsarine Catherine II de Russie, très au fait de la position de Diderot dans l'intelligentsia parisienne. On peut voir aujourd'hui au musée de l'Ermitage à Saint-Pétersbourg nombre de tableaux dont il a été question dans les Salons rédigés par Diderot. D'autres correspondances parisiennes bâties sur le même modèle et pour le même type d'abonnés friands des modes françaises concurrençaient celle de Grimm ; la plupart étaient le fait d'agents des princes allemands à Paris, ce qu'était d'ailleurs Grimm pour le duc de Saxe-Gotha ${ }^{122}$. Certains nouvellistes à la main parisiens pratiquaient depuis longtemps pour leurs abonnés

121 Divers comptes rendus imprimés en étaient faits dans la presse et dans des brochures spécialisées, à côté de simples « livrets » de salon informatifs (Hélène Zmijewska, «La critique des salons en France avant Diderot », Gazette des beaux-arts, juillet-août 1970, p. 1-44). 
français ces « commissions " qui allaient des achats de produits de luxe aux placements financiers ${ }^{123}$. La presse moderne, organisée autour de la publicité et financée en grande partie par elle, avait donc connu dès le XVIII ${ }^{\mathrm{e}}$ siècle une ébauche où l'on trouvait aussi les petites annonces que semble avoir inaugurées en 1717 le Nouveau Mercure ${ }^{124}$.

Ce rapide survol de la presse de langue française dans le dernier siècle de l'Ancien Régime, avant les bouleversements de la Révolution ${ }^{125}$, montre comment la communication périodique passa d'un strict contrôle étatique à une diversification qui allait de pair avec la critique, opérée par l'opinion publique, de la légitimité des acteurs qui s'en étaient arrogé le monopole. Il s'avérait illusoire d'imposer la " voix de la France » à un lectorat qui pouvait avoir accès aux gazettes et aux journaux étrangers, aux nouvelles à la main et aux brochures clandestines qui allaient des chansons satiriques aux projets de réforme plus ou moins utopiques. Tout un monde d'écrivains de contrebande, dont Robert Darnton a montré l'importance dans la formation de l'esprit contestataire des dernières décennies du XVIII ${ }^{\mathrm{e}}$ siècle ${ }^{126}$, s'agitait à Paris, en province et à l'étranger pour démentir la Gazette de France Mais la presse moderne était déjà en germe dans l'évolution qu'elle connut vers la concentration capitaliste (Panckoucke), la professionnalisation des journalistes et la concurrence des titres.

\section{FRANÇOIS MOUREAU}

Université Paris-Sorbonne

123 Répertoire des nouvelles à la main, " Préface », p. XXVIII.

124 Dictionnaire des journaux, $\mathrm{n}^{\circ}$ 922. Au XVII ${ }^{\mathrm{e}}$ siècle, la Feuille du Bureau d'Adresse de Théophraste Renaudot (Dictionnaire des journaux, $\mathrm{n}^{\circ} 457$ ) est une première tentative de journal d'annonces publicitaires.

125 Pierre Rétat, Les Journaux de 1789. Bibliographie critique, Paris, Éditions du CNRS, 1988 ; Claude Labrosse et Pierre Rétat, Naissance du journal révolutionnaire, Presses universitaires de Lyon, 1989 ; Christophe Cave, Denis Reynaud, Danièle Willemart, avec la collaboration de Henri Duranton, 1793 : L'esprit des journaux, Publications de l'Université de Saint-Étienne, 1993.

126 Bohème littéraire et Révolution : le monde des livres au XVIII siècle, Paris, GallimardLe Seuil, 1983 ; Édition et sédition. L'univers de la littérature clandestine au XVIII siècle, Paris, Gallimard, 1991 ; The Corpus of Clandestine Literature in France 1769-1789, New York, London, W. W. Norton, 1995; The Forbidden Best-Sellers of Pre-Revolutionary France, London, Harper-Collins, 1996. 